\title{
SMIRNOV'S INEQUALITY FOR POLYNOMIALS HAVING ZEROS OUTSIDE THE UNIT DISC
}

\begin{abstract}
In 1887, the famous chemist D. I. Mendeleev posed the following problem: to estimate $\left|f^{\prime}(x)\right|$ for a real polynomial $f(x)$, satisfying the condition $|f(x)| \leqslant M$ on $[a, b]$. This question arose when Mendeleev was studying aqueous solutions. The problem was solved by the famous mathematician A. A. Markov, and over the following 100 years was repeatedly modified and extended. For complex polynomials, important inequalities were obtained by $\mathrm{S}$. N. Bernstein and V. I. Smirnov. Many other well-known mathematicians, such as Ch. Pommerenke, G. Szegö, Q. I. Rahman, G. Schmeisser, worked in this subject. Almost all results in this direction significantly use the following condition: all zeros of a majorizing polynomial belong to the closed unit disc. In this paper, we remove this condition. Here a majorizing polynomial may have zeros outside the unit disc. This allows to extend the inequalities of Bernstein and Smirnov.
\end{abstract}

Key words: polynomial, the Smirnov inequality, the Bernstein inequality

2020 Mathematical Subject Classification: 30C10, 30A10

1. Introduction. The subject discussed in this article can be traced back to the problem posed by the famous chemist D. I. Mendeleev in 1887: for a polynomial $f$, $\operatorname{deg} f=2$, with $|f(x)| \leqslant M$ for $x \in[a, b]$, give an estimate for $\left|f^{\prime}(x)\right|$ on $[a, b][12, \S 86]$. Over the following 100 years, the Mendeleev problem was repeatedly modified and extended. For example, in the book by V. I. Smirnov and N. A. Lebedev [16, p. 340], this problem was formulated in a more general form:

Let $B \subset \mathbb{C}$ be a compact set, $f(z)$ be a polynomial with $\operatorname{deg} f=n \geqslant 1$, and suppose that $|f(z)| \leqslant M$ for all $z \in B$. Give an estimate for $\left|f^{\prime}(z)\right|$ for $z \in B$.

(C) Petrozavodsk State University, 2021 
The original problem posed by Mendeleev [12, § 86] was solved by A. A. Markov in 1889 for a polynomial $f$ with $\operatorname{deg} f=n$.

Theorem A. [9], [10, p. 51-75]. Let $f$ be a polynomial with $\operatorname{deg} f=n$ and suppose that $|f(x)| \leqslant M$ on $[-1,1]$. Then, for $x \in[-1,1]$ :

$$
\left|f^{\prime}(x)\right| \leqslant n^{2} M
$$

Equality is attained only for the functions

$$
f(x)= \pm M T_{n}(x)
$$

where $T_{n}(x)=\cos (n \arccos x)$ are the Chebyshev polynomials.

Inequality (1) was refined by S. N. Bernstein in 1912 in the following way:

Theorem B. [1, p. 25]. Let $f$ be a polynomial from Theorem A. Then, for $x \in[-1,1]$

$$
\left|f^{\prime}(x)\right| \leqslant \frac{n M}{\sqrt{1-x^{2}}} .
$$

Theorem B was of a considerable use for answering the following question of the best approximation: is it possible to approximate the function $|x|$ by polynomials of degree $n$ with an error $o(1 / n)$ as $n \rightarrow \infty$ ? This question was posted in 1903 as a competition by the Belgian Academy of Science on a suggestion by Ch. J. de Vallée-Poussin. S. N. Bernstein gave the negative answer to this question in [1], which was published by the Belgian Academy as the prize-winning treatise. For more mathematical and historical details see, for example, [6, p. 157-161], [17, p. 182-186].

V. A. Markov obtained a result analogous to Theorem A for the $k$-th derivative of a polynomial $f, 1 \leqslant k \leqslant n$, [11]. Note that Mendeleev and Markovs dealt only with real polynomials. For more details concerning this question, we also refer the reader to the historical survey in [7].

In this paper, we consider complex polynomials. Let $\mathbb{D}$ denote the unit disc $\{z \in \mathbb{C}:|z|<1\}$. V. I. Smirnov found the following solution to the Mendeleev problem for complex polynomials with $B=\partial \mathbb{D}$ :

Theorem C. [15], [16, ch.V, $\S 1,2^{0}$, p. 346] Let $f$ be a polynomial with $\operatorname{deg} f=n$ and suppose that $|f(z)| \leqslant M$ on $\partial \mathbb{D}$. Then, for $z \in \partial \mathbb{D}$ :

$$
\left|f^{\prime}(z)\right| \leqslant M n \text {. }
$$


Equality holds only if $f(z)=e^{i \gamma} z^{n}, \gamma \in \mathbb{R}$.

Similar results have been obtained for some other classes of compact sets $B \subset \mathbb{C}$, for example, by Ch. Pommerenke [13] and G. Szegö [18] (see also [16, pp. 351, 352]). We shall not dwell on these results, since we are interested in the case $B=\partial \mathbb{D}$ only.

It is convenient to rewrite Theorem $\mathrm{C}$ as follows:

Theorem $\mathbf{C}^{\prime}$. Let $f$ be a polynomial with $\operatorname{deg} f=n$ and suppose that $|f(z)| \leqslant\left|M z^{n}\right|$ on $\partial \mathbb{D}$. Then

$$
\left|f^{\prime}(z)\right| \leqslant\left|\left(M z^{n}\right)^{\prime}\right|, \quad z \in \partial \mathbb{D} .
$$

Equality holds only if $f(z)=e^{i \gamma} z^{n}, \gamma \in \mathbb{R}$.

In 1930, S. N. Bernstein obtained a generalization of Theorem $\mathrm{C}^{\prime}$, replacing the polynomial $M z^{n}$ with an arbitrary polynomial $F$ of degree $n$, and obtained the following result:

Theorem D. [2] (see also [3, p. 497], [14, p. 510]) Let $f$ and $F$ be polynomials, such that:

1) $\operatorname{deg} f \leqslant \operatorname{deg} F=n$,

2) $|f(z)| \leqslant|F(z)|$ on $\partial \mathbb{D}$,

3) $F$ has all its zeros in $\overline{\mathbb{D}}$.

Then

$$
\left|f^{\prime}(z)\right| \leqslant\left|F^{\prime}(z)\right| \quad \text { for } z \in \mathbb{C} \backslash \mathbb{D} .
$$

For $z \in \mathbb{C} \backslash \overline{\mathbb{D}}$, equality holds only for $f=e^{i \gamma} F, \gamma \in \mathbb{R}$.

Let $R \geqslant 1$; denote by $\Omega_{R}$ the image of the $\operatorname{disc}\{z \in \mathbb{C}:|z| \leqslant R\}$ under the mapping $\psi(z)=t(1+t)^{-1}$. For a polynomial $f$ of degree at most $n$ put

$$
S_{\alpha}[f](z)=z f^{\prime}(z)-n \alpha f(z)
$$

where $\alpha$ is a complex constant.

V. I. Smirnov gave the following generalization of Theorem D:

Theorem E. [16, ch. V, $\S 1$, p. 356] Let $R \geqslant 1, f$ and $F$ be polynomials from Theorem $D$. Then, for $|z|=R$,

$$
\left|S_{\alpha}[f](z)\right| \leqslant\left|S_{\alpha}[F](z)\right|
$$

for all $\alpha \in \Omega_{R}$. 
For $\alpha \in \operatorname{int} \Omega_{R}$ and $z \in \mathbb{C} \backslash \overline{\mathbb{D}}$, equality in (3) holds only for $f=e^{i \gamma} F$, $\gamma \in \mathbb{R}$

From the maximum modulus principle it follows that it is possible to replace condition 2) in Theorem $\mathrm{D}$ and Theorem $\mathrm{E}$ by the equivalent condition: $|f(z)| \leqslant|F(z)|$ for $z \in \mathbb{C} \backslash \mathbb{D}$.

Putting $\alpha=0$ in Theorem E, we obtain Theorem D. From Theorem D and Theorem E, we see that the differentiation operator and the Smirnov differential operator $S_{\alpha}$ preserve inequality between polynomials. Saying this, we mean the following: the inequality $|f(z)| \leqslant|F(z)|$ on $\partial \mathbb{D}$ remains valid if we apply one of these operators to polynomials $f$ and $F$, with the conditions of Theorem D satisfied. Such type of operators was widely investigated (see, for example, [14], [4], [5], [19] and references therein). Almost all results concerning such operators significantly use condition 3) from Theorem D. We waived this condition allowing the polynomial $F$ to have one zero outside the unit disc (see [8]): we proved the following

Theorem F. [8] Let $R \geqslant 1$. Let $f$ and $F$ be polynomials, such that

1) $\operatorname{deg} f \leqslant \operatorname{deg} F=n$,

2) $|f(z)| \leqslant|F(z)|$ for $z \in \mathbb{C} \backslash \mathbb{D}$,

3) $z_{0}$ is a unique zero of $F$ lying in $\mathbb{C} \backslash \overline{\mathbb{D}}, k$ is order of $z_{0}, 1 \leqslant k \leqslant n-1$.

Then

$$
\left|S_{\alpha}[f](z)\right| \leqslant\left|S_{\alpha}[F](z)\right|
$$

for $|z|=R$ and for $\alpha \in D_{R}$, where $D_{R}$ is one of the sets

a) complement to the disc

$\left\{\alpha \in \mathbb{C}:\left|\alpha-\frac{n-k}{n} \frac{R^{2}}{R^{2}-1}-\frac{R^{2}}{n} \frac{k}{R^{2}-\left|z_{0}\right|^{2}}\right|<\frac{n-k}{n} \frac{R}{R^{2}-1}+\frac{R}{n} \frac{k\left|z_{0}\right|}{\left.\left|R^{2}-\right| z_{0}\right|^{2} \mid}\right\}$

if $R>1$ and $\left|z_{0}\right| \neq R$;

b) complement to the strip

$$
\left\{\alpha \in \mathbb{C}:\left|\operatorname{Re} \alpha-\frac{n-k}{n} \frac{R^{2}}{R^{2}-1}-\frac{k}{2 n}\right|<\frac{n-k}{n} \frac{R}{R^{2}-1}\right\}
$$

if $R>1,\left|z_{0}\right|=R$;

c) the half-plane

$$
\left\{\alpha \in \mathbb{C}: \operatorname{Re} \alpha \leqslant \frac{k}{n}\left(\frac{1}{1-\left|z_{0}\right|}+\frac{1}{2}\left(1-\frac{k}{n}\right)\right)\right\}
$$


if $R=1$.

For $z=z_{0}, k>1$, equality in (4) holds for every $\alpha \in \mathbb{C}$ and every $f$ and $F$ satisfying conditions of Theorem $F$.

For the case $z=z_{0}, k=1, \alpha \in \mathbb{C}$ and the case $z \neq z_{0}, z \in \mathbb{C} \backslash \overline{\mathbb{D}}$, $\alpha \in$ int $D_{R}$, in (4) equality holds only if $f=e^{i \gamma} F, \gamma \in \mathbb{R}$.

In this paper, we consider the case when the polynomial $F$ has an arbitrary number of zeros outside the closed unit disc.

\section{Refinement of the Smirnov inequality.}

Theorem 1. Suppose that $R \geqslant 1$. Let $f$ and $F$ be polynomials, such that

1) $\operatorname{deg} f \leqslant \operatorname{deg} F=n$,

2) $|f(z)| \leqslant|F(z)|$ for $z \in \mathbb{C} \backslash \mathbb{D}$,

3) $z_{1}, \ldots, z_{p}$ are all the zeros of $F$ lying in $\mathbb{C} \backslash \overline{\mathbb{D}} ; k_{1}, \ldots, k_{p}$ are their orders, correspondingly, $1 \leqslant k=k_{1}+\cdots+k_{p} \leqslant n-1$.

Then

$$
\left|S_{\alpha}[f](z)\right| \leqslant\left|S_{\alpha}[F](z)\right|
$$

for $|z|=R$ and for $\alpha \in D(R, p, n)$, where $D(R, p, n)$ is one of the sets:

a) complement to the disc

$$
\left\{\alpha \in \mathbb{C}:\left|\alpha-c_{p}\right|<r_{p}\right\}
$$

where

$$
\begin{aligned}
& c_{p}=\frac{n-k}{n} \frac{R^{2}}{R^{2}-1}+\frac{R^{2}}{n} \sum_{j=1}^{p} \frac{k_{j}}{R^{2}-\left|z_{j}\right|^{2}}, \\
& r_{p}=\frac{n-k}{n} \frac{R}{R^{2}-1}+\frac{R}{n} \sum_{j=1}^{p} \frac{k_{j}\left|z_{j}\right|}{\left.\left|R^{2}-\right| z_{j}\right|^{2} \mid},
\end{aligned}
$$

if $R>1$ and all the zeros $z_{1}, \ldots, z_{p}$ do not belong to the circle $|z|=R$;

b) complement to the strip

$$
\left\{\alpha \in \mathbb{C}:\left|\operatorname{Re} \alpha-s_{p}\right|<\frac{n-k}{n} \frac{R}{R^{2}-1}\right\}
$$

where

$$
s_{p}=\frac{n-k}{n} \frac{R^{2}}{R^{2}-1}+\frac{k}{2 n},
$$


if $R>1$ and $\left|z_{1}\right|=\cdots=\left|z_{p}\right|=R$;

\section{c) complement to the strip}

$$
\left\{\alpha \in \mathbb{C}:\left|\operatorname{Re} \alpha-x_{q, p}\right|<\rho_{q, p}\right\},
$$

where

$$
\begin{gathered}
x_{q, p}=\frac{n-k}{n} \frac{R^{2}}{R^{2}-1}+\frac{R^{2}}{n} \sum_{j=1}^{q} \frac{k_{j}}{R^{2}-\left|z_{j}\right|^{2}}+\frac{1}{2 n} \sum_{j=q+1}^{p} k_{j}, \\
\rho_{q, p}=\frac{n-k}{n} \frac{R}{R^{2}-1}+\frac{R}{n} \sum_{j=1}^{q} \frac{k_{j}\left|z_{j}\right|}{\left.\left|R^{2}-\right| z_{j}\right|^{2} \mid},
\end{gathered}
$$

if $R>1,\left|z_{1}\right|, \ldots,\left|z_{q}\right| \neq R ;\left|z_{q+1}\right|=\ldots=\left|z_{p}\right|=R ; q \in \mathbb{N}, 1 \leqslant k_{1}+\ldots+k_{q} \leqslant n-2$;

d) the half-plane

$$
\left\{\alpha \in \mathbb{C}: \operatorname{Re} \alpha \leqslant \frac{1}{n} \sum_{j=1}^{p} \frac{k_{j}}{1-\left|z_{j}\right|}+\frac{1}{2}\left(1-\frac{k}{n}\right)\right\}
$$

if $R=1$.

For $z=z_{j}, j=1, \ldots, p, k_{j}>1$ in (5), equality holds for every $\alpha \in \mathbb{C}$ and all pairs $f$ and $F$ satisfying the theorem conditions.

If $z=z_{j}, j=1, \ldots, p, k_{j}=1, \alpha \in \mathbb{C}$ or $z \in \mathbb{C} \backslash \overline{\mathbb{D}}, z \neq z_{j}, j=1, \ldots, p$, $\alpha \in \operatorname{int} D(R, p, n)$, then equality in (5) takes place only if $f=e^{i \gamma} F, \gamma \in \mathbb{R}$.

Remark. From the proof of Theorem 1, we see that item a) does not describe the whole set of values of $\alpha$, for which (5) is true. The set $D(R, p, n)$ from item a) can be extended, but this extension requires much more complicated description.

Proof. By condition 3) of Theorem 1, we see that the polynomial $F$ can be written in the form

$$
F(z)=\left(z-z_{1}\right)^{k_{1}} \ldots\left(z-z_{p}\right)^{k_{p}} F_{1}(z)
$$

here $F_{1}(z) \neq 0$ in $\mathbb{C} \backslash \overline{\mathbb{D}}$. By condition 2$)$, the polynomial $f$ has the representation

$$
f(z)=\left(z-z_{1}\right)^{k_{1}} \ldots\left(z-z_{p}\right)^{k_{p}} f_{1}(z) .
$$


We prove items a), b), and d) of Theorem 1 by mathematical induction. For $p=1$, we obtain the statement of Theorem $\mathrm{F}$.

Suppose that the theorem statement is true for $p-1,1 \leqslant k_{1}+\ldots+$ $+k_{p-1} \leqslant n-2$. Prove that Theorem 1 is valid for $p, 2 \leqslant k=k_{1}+\ldots+k_{p} \leqslant n-1$.

Denote (see (6) and (7))

$$
\begin{aligned}
& F_{2}(z)=\left(z-z_{1}\right)^{k_{1}} \ldots\left(z-z_{p-1}\right)^{k_{p-1}} F_{1}(z), \\
& f_{2}(z)=\left(z-z_{1}\right)^{k_{1}} \ldots\left(z-z_{p-1}\right)^{k_{p-1}} f_{1}(z) .
\end{aligned}
$$

Then $F(z)=\left(z-z_{p}\right)^{k_{p}} F_{2}(z), f(z)=\left(z-z_{p}\right)^{k_{p}} f_{2}(z)$, and, by item 2) of Theorem $1,\left|f_{2}(z)\right| \leqslant\left|F_{2}(z)\right|,|z| \geqslant 1$.

Let us find the set of constants $\alpha$, such that (5) takes place for all $z$ on the circle $|z|=R$. Write (5) as

$$
\left|z f^{\prime}(z)-\alpha n f(z)\right| \leqslant\left|z F^{\prime}(z)-\alpha n F(z)\right|, \quad|z|=R,
$$

or

$$
\begin{aligned}
& \left|z\left(k_{p}\left(z-z_{p}\right)^{k_{p}-1} f_{2}(z)+\left(z-z_{p}\right)^{k_{p}} f_{2}^{\prime}(z)\right)-\alpha n\left(z-z_{p}\right)^{k_{p}} f_{2}(z)\right| \leqslant \\
\leqslant & \left|z\left(k_{p}\left(z-z_{p}\right)^{k_{p}-1} F_{2}(z)+\left(z-z_{p}\right)^{k_{p}} F_{2}^{\prime}(z)\right)-\alpha n\left(z-z_{p}\right)^{k_{p}} F_{2}(z)\right| .
\end{aligned}
$$

For $z=z_{p}$, (8) takes place for all $\alpha \in \mathbb{C}$. If $z=z_{p}$ and $k_{p}>1$, then equality in (8) holds for every $f_{2}$ and $F_{2}$. Therefore, equality in (5) takes place for every pair of the polynomials $f$ and $F$ satisfying the conditions of the theorem.

If $z=z_{p}$ and $k_{p}=1$, then, by (8), equality in (5) holds only if $f(z)=$ $=e^{i \gamma} F(z), \gamma \in \mathbb{R}$.

Obviously, we can change the index $p$ with any $j, j=1, \ldots, p-1$, arguing as above about equality in (5).

Assuming, further, $z \neq z_{p}$, we rewrite (8) in the form

$$
\left|z f_{2}^{\prime}(z)-\left(\alpha n-k_{p} \frac{z}{z-z_{p}}\right) f_{2}(z)\right| \leqslant\left|z F_{2}^{\prime}(z)-\left(\alpha n-k_{p} \frac{z}{z-z_{p}}\right) F_{2}(z)\right|,|z|=R \text {. }
$$

By the induction assumption, Theorem 1 is true for the polynomials $f_{2}$ and $F_{2}$ with $n$ replaced by $n-k_{p}$. Hence, we can rewrite (9) as

$$
\left|S_{\beta}\left[f_{2}\right](z)\right| \leqslant\left|S_{\beta}\left[F_{2}\right](z)\right|, \quad|z|=R,
$$

where

$$
\beta=\frac{1}{n-k_{p}}\left(\alpha n-k_{p} \frac{z}{z-z_{p}}\right) \in D\left(R, p-1, n-k_{p}\right) .
$$


Then

$$
\alpha=\left(1-\frac{k_{p}}{n}\right) \beta+\frac{k_{p}}{n} \frac{z}{z-z_{p}}, \quad|z|=R,
$$

and for a fixed $z,|z|=R$, the set $G_{z}$ of values of $\alpha$ is the set $\left(1-\frac{k_{p}}{n}\right) D\left(R, p-1, n-k_{p}\right)$ shifted by $\frac{k_{p}}{n} \frac{z}{z-z_{p}}$. Therefore, the set of the values $\alpha$, such that (5) is true, is the set $\bigcap_{|z|=R} G_{z}$.

First, we consider the case $\mathbf{R}>\mathbf{1}$.

a) Let $\left|\mathbf{z}_{\mathbf{j}}\right| \neq \mathbf{R}$ for all $\mathbf{j}=\mathbf{1}, \ldots, \mathbf{p}$. By the inductive assumption, inequality (10) is true for $\beta$ that belong to $D\left(R, p-1, n-k_{p}\right)$. This set is the complement to the open disc

$$
C=\left\{\beta \in \mathbb{C}:\left|\beta-c_{p-1}\right|<r_{p-1}\right\},
$$

where

$$
\begin{aligned}
& c_{p-1}=\frac{\left(n-k_{p}\right)-k_{1}-\cdots-k_{p-1}}{n-k_{p}} \frac{R^{2}}{R^{2}-1}+\frac{R^{2}}{n-k_{p}} \sum_{j=1}^{p-1} \frac{k_{j}}{R^{2}-\left|z_{j}\right|^{2}}, \\
& r_{p-1}=\frac{\left(n-k_{p}\right)-k_{1}-\cdots-k_{p-1}}{n-k_{p}} \frac{R}{R^{2}-1}+\frac{R}{n-k_{p}} \sum_{j=1}^{p-1} \frac{k_{j}\left|z_{j}\right|}{\left.\left|R^{2}-\right| z_{j}\right|^{2} \mid} .
\end{aligned}
$$

The function $\frac{z}{z-z_{p}}$ maps $|z|=R$ to the circle with center $\frac{R^{2}}{R^{2}-\left|z_{p}\right|^{2}}$ and radius $\frac{R\left|z_{p}\right|}{\left.\left|R^{2}-\right| z_{p}\right|^{2} \mid}$. Hence, the set of all centers of the discs

$$
C_{z}=\left(1-\frac{k_{p}}{n}\right) C+\frac{k_{p}}{n} \frac{z}{z-z_{p}},|z|=R,
$$

is the circle with center

$$
\begin{gathered}
\left(1-\frac{k_{p}}{n}\right) c_{p-1}+\frac{k_{p}}{n} \frac{R^{2}}{R^{2}-\left|z_{p}\right|^{2}}= \\
=\frac{n-k_{p}}{n}\left(\frac{n-k}{n-k_{p}} \frac{R^{2}}{R^{2}-1}+\frac{R^{2}}{n-k_{p}} \sum_{j=1}^{p-1} \frac{k_{j}}{R^{2}-\left|z_{j}\right|^{2}}\right)+\frac{k_{p}}{n} \frac{R^{2}}{R^{2}-\left|z_{p}\right|^{2}}= \\
=\frac{n-k}{n} \frac{R^{2}}{R^{2}-1}+\frac{R^{2}}{n} \sum_{j=1}^{p} \frac{k_{j}}{R^{2}-\left|z_{j}\right|^{2}}=c_{p}
\end{gathered}
$$


and radius $\frac{k_{p}}{n} \frac{R\left|z_{p}\right|}{\left.\left|R^{2}-\right| z_{p}\right|^{2} \mid}$. Note that

$$
\bigcap_{|z|=R} G_{z}=\bigcap_{|z|=R}\left(\mathbb{C} \backslash C_{z}\right)
$$

contains the complement to the disc

$$
\left\{\alpha \in \mathbb{C}:\left|\alpha-c_{p}\right|<A\right\},
$$

where

$$
\begin{gathered}
A=\frac{k_{p}}{n} \frac{R\left|z_{p}\right|}{\left.\left|R^{2}-\right| z_{p}\right|^{2} \mid}+\left(1-\frac{k_{p}}{n}\right) r_{p-1}= \\
=\frac{k_{p}}{n} \frac{R\left|z_{p}\right|}{\left.\left|R^{2}-\right| z_{p}\right|^{2} \mid}+\frac{n-k_{p}}{n}\left(\frac{n-k}{n-k_{p}} \frac{R}{R^{2}-1}+\frac{R}{n-k_{p}} \sum_{j=1}^{p-1} \frac{k_{j}\left|z_{j}\right|}{\left.\left|R^{2}-\right| z_{j}\right|^{2} \mid}\right)= \\
=\frac{n-k}{n} \frac{R}{R^{2}-1}+\frac{R}{n} \sum_{j=1}^{p} \frac{k_{j}\left|z_{j}\right|}{\left.\left|R^{2}-\right| z_{j}\right|^{2} \mid}=r_{p} .
\end{gathered}
$$

Hence, $D(R, p, n) \subset \bigcap_{|z|=R} G_{z}$ and a $)$ is proved.

b) Let $\left|\mathbf{z}_{\mathbf{j}}\right|=\mathbf{R}$ for all $\mathbf{j}=\mathbf{1}, \ldots, \mathbf{p}$. By the induction assumption, $\beta$ belongs to the set

$D\left(R, p-1, n-k_{p}\right)=\left\{\beta \in \mathbb{C}:\left|\operatorname{Re} \beta-s_{p-1}\right| \geqslant \frac{\left(n-k_{p}\right)-k_{1}-\ldots-k_{p-1}}{n-k_{p}} \frac{R}{R^{2}-1}\right\}$,

where

$$
s_{p-1}=\frac{\left(n-k_{p}\right)-k_{1}-\cdots-k_{p-1}}{n-k_{p}} \frac{R^{2}}{R^{2}-1}+\frac{k_{1}+\cdots+k_{p-1}}{2\left(n-k_{p}\right)} .
$$

The function $\frac{z}{z-z_{p}}$ maps the circle $|z|=R$ to the straight line $\operatorname{Re} z=\frac{1}{2}$. Therefore, in this case we have

$$
D(R, p, n)=\bigcap_{|z|=R} G_{z}=\left(1-\frac{k_{p}}{n}\right) D\left(R, p-1, n-k_{p}\right)+\frac{k_{p}}{2 n} .
$$

This implies that $D(R, p, n)=\{\alpha \in \mathbb{C}:|\operatorname{Re} \alpha-B| \geqslant D\}$, where

$$
B=\left(1-\frac{k_{p}}{n}\right) s_{p-1}+\frac{k_{p}}{2 n}=
$$


$=\frac{n-k_{p}}{n}\left(\frac{n-k}{n-k_{p}} \frac{R^{2}}{R^{2}-1}+\frac{k_{1}+\ldots+k_{p-1}}{2\left(n-k_{p}\right)}\right)+\frac{k_{p}}{2 n}=\frac{n-k}{n} \frac{R^{2}}{R^{2}-1}+\frac{k}{2 n}=s_{p}$

and

$$
D=\left(1-\frac{k_{p}}{n}\right) \frac{n-k}{n-k_{p}} \frac{R}{R^{2}-1}=\frac{n-k}{n} \frac{R}{R^{2}-1} .
$$

d) Consider the case $\mathbf{R}=\mathbf{1}$. By the induction assumption, $\beta$ belongs to the half-plane

$$
\begin{aligned}
& D\left(R, p-1, n-k_{p}\right)= \\
& \quad=\left\{\beta \in \mathbb{C}: \operatorname{Re} \beta \leqslant \frac{1}{n-k_{p}} \sum_{j=1}^{p-1} \frac{k_{j}}{1-\left|z_{j}\right|}+\frac{1}{2}\left(1-\frac{k_{1}+\cdots+k_{p-1}}{n-k_{p}}\right)\right\} .
\end{aligned}
$$

Since the function $\frac{z}{z-z_{p}}$ maps $\partial \mathbb{D}$ to the circle with diameter $\left[\frac{1}{1-\left|z_{p}\right|}, \frac{1}{1+\left|z_{p}\right|}\right]$, we learn that the parameter $\alpha$ (see (12)) belongs to the set

$$
D(1, p, n)=\bigcap_{|z|=1} G_{z}
$$

where

$$
G_{z}=\left(1-\frac{k_{p}}{n}\right) D\left(1, p-1, n-k_{p}\right)+\frac{k_{p}}{n} \frac{z}{z-z_{p}} .
$$

This intersection is the set $D(1, p, n)=\{\alpha \in \mathbb{C}: \operatorname{Re} \alpha \leqslant E\}$, where

$$
\begin{gathered}
E=\left(1-\frac{k_{p}}{n}\right)\left[\frac{1}{n-k_{p}} \sum_{j=1}^{p-1} \frac{k_{j}}{1-\left|z_{j}\right|}+\frac{1}{2}\left(1-\frac{k_{1}+\cdots+k_{p-1}}{n-k_{p}}\right)\right]+\frac{k_{p}}{n} \frac{1}{1-\left|z_{p}\right|}= \\
=\frac{1}{n} \sum_{j=1}^{p} \frac{k_{j}}{1-\left|z_{j}\right|}+\frac{1}{2}\left(1-\frac{k}{n}\right) .
\end{gathered}
$$

Let us consider separately the case $\mathbf{c}$ ) when $\mathbf{R}>\mathbf{1}, \mathbf{z}_{\mathbf{1}}, \ldots, \mathbf{z}_{\mathbf{q}}$ do not lie on the circle $|\mathbf{z}|=\mathbf{R}$, but $\left|\mathbf{z}_{\mathbf{q}+\mathbf{1}}\right|=\ldots=\left|\mathbf{z}_{\mathbf{p}}\right|=\mathbf{R}, 1 \leqslant k_{1}+\ldots+k_{q} \leqslant n-2$, $q \in \mathbb{N}$.

Apply the proved item a) of Theorem 1 to the polynomials

$$
\widetilde{F}_{q}(z)=\left(z-z_{1}\right)^{k_{1}} \ldots\left(z-z_{q}\right)^{k_{q}} F_{1}(z), \quad \widetilde{f}_{q}(z)=\left(z-z_{1}\right)^{k_{1}} \ldots\left(z-z_{q}\right)^{k_{q}} f_{1}(z),
$$


where $f_{1}$ and $F_{1}$ are the polynomials from representations (6) and (7). We obtain

$$
\left|S_{\alpha_{q}}\left[\widetilde{f}_{q}\right](z)\right| \leqslant\left|S_{\alpha_{q}}\left[\widetilde{F}_{q}\right](z)\right|
$$

for $|z|=R$ and $\alpha_{q}$ from the set $D\left(R, q, n-k_{q+1}-\cdots-k_{p}\right)$. This set is the complement to the $\operatorname{disc}\left\{\alpha_{q} \in \mathbb{C}:\left|\alpha_{q}-c_{q}\right|<r_{q}\right\}$. Consider the polynomials

$$
\widetilde{F}_{q+1}(z)=\left(z-z_{q+1}\right)^{k_{q+1}} \widetilde{F}_{q}(z), \quad \widetilde{f}_{q+1}(z)=\left(z-z_{q+1}\right)^{k_{q+1}} \widetilde{f}_{q}(z) .
$$

Similarly to how we previously rewrote inequality (5) in the form (10), we rewrite the inequality

$$
\left|S_{\alpha_{q+1}}\left[\widetilde{f}_{q+1}\right](z)\right| \leqslant\left|S_{\alpha_{q+1}}\left[\widetilde{F}_{q+1}\right](z)\right|, \quad|z|=R,
$$

in form (13), changing $n$ to $n-k_{q+2}-\cdots-k_{p}$. Similarly to (12), the parameters $\alpha_{q+1}$ and $\alpha_{q}$ are connected by the following relationship:

$$
\alpha_{q+1}=\left(1-\frac{k_{q+1}}{n-k_{q+2}-\cdots-k_{p}}\right) \alpha_{q}+\frac{k_{q+1}}{n-k_{q+2}-\cdots-k_{p}} \frac{z}{z-z_{q+1}} .
$$

Since the function $\frac{z}{z-z_{q+1}}$ maps the circle $|z|=R$ to the straight line $\operatorname{Re} z=\frac{1}{2}$ and $\alpha_{q} \in D\left(R, q, n-k_{q+1}-\cdots-k_{p}\right)$, we learn that $\alpha_{q+1}$ belongs to the set

$$
\left\{\alpha_{q+1} \in \mathbb{C}:\left|\operatorname{Re} \alpha_{q+1}-H\right| \geqslant I\right\}
$$

where

$$
\begin{gathered}
H=\left(1-\frac{k_{q+1}}{n-k_{q+2}-\cdots-k_{p}}\right) c_{q}+\frac{k_{q+1}}{n-k_{q+2}-\cdots-k_{p}} \frac{1}{2}= \\
=\frac{n-k_{q+1}-\cdots-k_{p}}{n-k_{q+2}-\cdots-k_{p}}\left(\frac{\left(n-k_{q+1}-\cdots-k_{p}\right)-k_{1}-\cdots-k_{q}}{n-k_{q+1}-\cdots-k_{p}} \frac{R^{2}}{R^{2}-1}+\right. \\
\left.+\frac{R^{2}}{n-k_{q+1}-\cdots-k_{p}} \sum_{j=1}^{q} \frac{k_{j}}{R^{2}-\left|z_{j}\right|^{2}}\right)+\frac{k_{q+1}}{n-k_{q+2}-\cdots-k_{p}} \frac{1}{2}= \\
=\frac{n-k}{n-k_{q+2}-\cdots-k_{p}} \frac{R^{2}}{R^{2}-1}+ \\
+\frac{R^{2}}{n-k_{q+2}-\cdots-k_{p}} \sum_{j=1}^{q} \frac{k_{j}}{R^{2}-\left|z_{j}\right|^{2}}+\frac{k_{q+1}}{n-k_{q+2}-\cdots-k_{p}} \frac{1}{2}=x_{q, q+1}
\end{gathered}
$$


and

$$
\begin{gathered}
I=\left(1-\frac{k_{q+1}}{n-k_{q+2}-\cdots-k_{p}}\right) r_{q}= \\
=\frac{n-k_{q+1}-\cdots-k_{p}}{n-k_{q+2}-\cdots-k_{p}}\left(\frac{\left(n-k_{q+1}-\cdots-k_{p}\right)-k_{1}-\cdots-k_{q}}{n-k_{q+1}-\cdots-k_{p}} \frac{R}{R^{2}-1}+\right. \\
\left.+\frac{R}{n-k_{q+1}-\cdots-k_{p}} \sum_{j=1}^{q} \frac{k_{j}\left|z_{j}\right|}{\left.\left|R^{2}-\right| z_{j}\right|^{2} \mid}\right)= \\
=\frac{R-k}{n-k_{q+2}-\ldots-k_{p}} \frac{R}{R^{2}-1}+\frac{R}{n-k_{q+2}-\ldots-k_{p}} \sum_{j=1}^{q} \frac{k_{j}\left|z_{j}\right|}{\left.\left|R^{2}-\right| z_{j}\right|^{2} \mid}=\rho_{q, q+1}
\end{gathered}
$$

in accordance with the definition of $\rho_{q, j}$ from the statement of Theorem 1. Therefore, we have proved item c) of Theorem 1 in the case when only one zero belongs to the circle $|z|=R$, i. e., $p=q+1$.

Further, we prove c) by mathematical induction. Suppose that c) takes place for the polynomials

$$
\begin{aligned}
& \widetilde{F}_{p-1}(z)=\left(z-z_{p-1}\right)^{k_{p-1}} \ldots\left(z-z_{1}\right)^{k_{1}} F_{1}(z), \\
& \widetilde{f}_{p-1}(z)=\left(z-z_{p-1}\right)^{k_{p-1}} \ldots\left(z-z_{1}\right)^{k_{1}} f_{1}(z) .
\end{aligned}
$$

Prove that c) is true for the polynomials

$$
F(z)=\left(z-z_{p}\right)^{k_{p}} \widetilde{F}_{p-1}(z), \quad f(z)=\left(z-z_{p}\right)^{k_{p}} \widetilde{f}_{p-1}(z) .
$$

As above, we rewrite (5) in the form

$$
\left|S_{\alpha_{p-1}}\left[\widetilde{f}_{p-1}\right](z)\right| \leqslant\left|S_{\alpha_{p-1}}\left[\widetilde{F}_{p-1}\right](z)\right|, \quad|z|=R
$$

with

$$
\alpha=\left(1-\frac{k_{p}}{n}\right) \alpha_{p-1}+\frac{k_{p}}{n} \frac{z}{z-z_{p}}, \quad|z|=R
$$

see (8)-(12) for details.

The function $\frac{z}{z-z_{p}}$ maps the circle $|z|=R$ to the straight line $\operatorname{Re} z=\frac{1}{2}$. By the assumption,

$$
\alpha_{p-1} \in D\left(R, p-1, n-k_{p}\right)=\left\{\alpha_{p-1} \in \mathbb{C}:\left|\operatorname{Re} \alpha_{p-1}-x_{q, p-1}\right| \geqslant \rho_{q, p-1}\right\} .
$$


Therefore, $\alpha$ belongs to the set

$$
\left(1-\frac{k_{p}}{n}\right) D\left(R, p-1, n-k_{p}\right)+\frac{k_{p}}{2 n}
$$

that is the set $\{\alpha \in \mathbb{C}:|\operatorname{Re} \alpha-J| \geqslant K\}$, where

$$
\begin{gathered}
J=\left(1-\frac{k_{p}}{n}\right) x_{q, p-1}+\frac{k_{p}}{2 n}= \\
=\frac{n-k_{p}}{n}\left(\frac{\left(n-k_{p}\right)-k_{1}-\cdots-k_{p-1}}{n-k_{p}} \frac{R^{2}}{R^{2}-1}+\right. \\
\left.+\frac{R^{2}}{n-k_{p}} \sum_{j=1}^{q} \frac{k_{j}}{R^{2}-\left|z_{j}\right|^{2}}+\frac{1}{2\left(n-k_{p}\right)} \sum_{j=q+1}^{p-1} k_{j}\right)+\frac{k_{p}}{2 n}= \\
=\frac{n-k}{n} \frac{R^{2}}{R^{2}-1}+\frac{R^{2}}{n} \sum_{j=1}^{q} \frac{k_{j}}{R^{2}-\left|z_{j}\right|^{2}}+\frac{1}{2 n} \sum_{j=q+1}^{p} k_{j}=x_{q, p}
\end{gathered}
$$

and

$$
\begin{gathered}
K=\left(1-\frac{k_{p}}{n}\right) \rho_{q, p-1}= \\
=\frac{n-k_{p}}{n}\left(\frac{\left(n-k_{p}\right)-k_{1}-\cdots-k_{p-1}}{n-k_{p}} \frac{R}{R^{2}-1} \frac{R}{n-k_{p}} \sum_{j=1}^{q} \frac{k_{j}\left|z_{j}\right|}{\left.\left|R^{2}-\right| z_{j}\right|^{2} \mid}\right)= \\
=\frac{n-k}{n} \frac{R}{R^{2}-1}+\frac{R}{n} \sum_{j=1}^{q} \frac{k_{j}\left|z_{j}\right|}{\left.\left|R^{2}-\right| z_{j}\right|^{2} \mid}=\rho_{q, p} .
\end{gathered}
$$

To prove Theorem 1, it remains to establish when (5) turns into equality for $z \in \mathbb{C} \backslash \overline{\mathbb{D}}, z \neq z_{j}, j=1, \ldots, p, \alpha \in \operatorname{int} D(R, p, n)$. For $p=1$, see Theorem F. Suppose that the theorem statement is true for $p-1$, $1 \leqslant k_{1}+\cdots+k_{p-1} \leqslant n-2$. For the mentioned $z$ and $\alpha \in \operatorname{int} D(R, p, n)$, (5) turns to equality if and only if (10) turns into equality for this $z$ and $\beta \in \operatorname{int} D\left(R, p-1, n-k_{p}\right)$. By the assumption, it is possible only in the case $f_{2}=e^{i \gamma} F_{2}, \gamma \in \mathbb{R}$. Thus, $f=e^{i \gamma} F$.

3. The Bernstein inequality inside the unit disc. As we have seen above, the classical Bernstein inequality (Theorem D) can be obtained from the Smirnov inequality (Theorem E), taking $\alpha=0$. However, we can not apply this reasoning to the polynomials with zeros in $\mathbb{C} \backslash \overline{\mathbb{D}}$, because 
the domains $D(R, p, n)$ from Theorem 1 do not always contain the point $\alpha=0$. Consequently, our first aim is to find a condition to guarantee that the point $\alpha=0$ belongs to the domain $D(R, p, n)$.

The case when the polynomial $F$ from Theorem 1 has a unique zero outside $\overline{\mathbb{D}}$ was considered in [8]. The following lemma was proved:

Lemma 1. [8] If the polynomial $F$ from Theorem 1 has a unique zero $z_{0}$ of order $k$ in $\mathbb{C} \backslash \overline{\mathbb{D}}, 1<R<\left|z_{0}\right|$, then the set $D(R, 1, n)$ contains the point $\alpha=0$ if and only if $R$ does not belong to the interval $\left(\left(1-\frac{k}{n}\right)\left|z_{0}\right|-\frac{k}{n} ;\left(1-\frac{k}{n}\right)\left|z_{0}\right|+\frac{k}{n}\right)$.

Now we consider the case when $F$ has an arbitrary number of zeros outside $\overline{\mathbb{D}}$.

Lemma 2. Let $F$ be the polynomial from Theorem $1, z_{1}, \ldots, z_{p}$ be all the zeros of $F$ in $\mathbb{C} \backslash \overline{\mathbb{D}}, 1<R<\min _{j=1, \ldots, p}\left|z_{j}\right|=\left|z_{p}\right|, k_{1} \ldots, k_{p}$ be orders of these zeros, correspondingly, $k=k_{1}+\cdots+k_{p}$. If

$$
R \leqslant\left(1-\frac{k}{n}\right)\left|z_{p}\right|-\frac{k}{n}
$$

then $0 \in D(R, p, n)$, where $D(R, p, n)$ is the domain from Theorem 1 .

Proof. Since $\left|z_{j}\right|>R>1$ for $j=1, \ldots, p$, we see that $D(R, p, n)$ is the domain from item a) of Theorem 1 . For $0 \in D(R, p, n)$, it is sufficient to prove that $\left|c_{p}\right| \geqslant r_{p}$, where $c_{p}$ and $r_{p}$ are the constants from item a) of Theorem 1.

Prove that $c_{p}>0$ under conditions of Lemma 2, i. e.,

$$
\frac{n-k}{n} \frac{R^{2}}{R^{2}-1}>\frac{R^{2}}{n} \sum_{j=1}^{p} \frac{k_{j}}{\left|z_{j}\right|^{2}-R^{2}}
$$

or

$$
\frac{n-k}{R^{2}-1}>\sum_{j=1}^{p} \frac{k_{j}}{\left|z_{j}\right|^{2}-R^{2}} .
$$

The last inequality follows from the inequality

$$
\frac{n-k}{R^{2}-1}>\sum_{j=1}^{p} \frac{k_{j}}{\left|z_{p}\right|^{2}-R^{2}}=\frac{k}{\left|z_{p}\right|^{2}-R^{2}}
$$


Inequality (15) is equivalent to

$$
n-k>\frac{R^{2}-1}{\left|z_{p}\right|^{2}-R^{2}} k=\left(\frac{\left|z_{p}\right|^{2}-1}{\left|z_{p}\right|^{2}-R^{2}}-1\right) k
$$

or

$$
n\left(\left|z_{p}\right|^{2}-R^{2}\right)>k\left(\left|z_{p}\right|^{2}-1\right)
$$

or

$$
\left|z_{p}\right|^{2}\left(1-\frac{k}{n}\right)+\frac{k}{n}>R^{2}
$$

To prove (16), rewrite (14) in the form

$$
R^{2} \leqslant\left(1-\frac{k}{n}\right)^{2}\left|z_{p}\right|^{2}-2 \frac{k}{n}\left(1-\frac{k}{n}\right)\left|z_{p}\right|+\frac{k^{2}}{n^{2}}
$$

Note that the left-hand side of (16) is greater than the right-hand side of (17):

$$
\left|z_{p}\right|^{2}\left(1-\frac{k}{n}\right)+\frac{k}{n}>\left(1-\frac{k}{n}\right)^{2}\left|z_{p}\right|^{2}-2 \frac{k}{n}\left(1-\frac{k}{n}\right)\left|z_{p}\right|+\frac{k^{2}}{n^{2}},
$$

because the last inequality is equivalent to

$$
\frac{k}{n}\left(1-\frac{k}{n}\right)\left|z_{p}\right|^{2}+2 \frac{k}{n}\left(1-\frac{k}{n}\right)\left|z_{p}\right|+\frac{k}{n}\left(1-\frac{k}{n}\right)>0
$$

or

$$
\left(\left|z_{p}\right|+1\right)^{2}>0 .
$$

Therefore, (16) is true, and we have proved that $c_{p}>0$.

It remains to show that $c_{p} \geqslant r_{p}$, i. e.,

$$
\frac{n-k}{n} \frac{R^{2}}{R^{2}-1}-\frac{R^{2}}{n} \sum_{j=1}^{p} \frac{k_{j}}{\left|z_{j}\right|^{2}-R^{2}} \geqslant \frac{n-k}{n} \frac{R}{R^{2}-1}+\frac{R}{n} \sum_{j=1}^{p} \frac{k_{j}\left|z_{j}\right|}{\left|z_{j}\right|^{2}-R^{2}} .
$$

Rewrite the last inequality in the equivalent form:

$$
\frac{n-k}{R+1} \geqslant \sum_{j=1}^{p} \frac{k_{j}}{\left|z_{j}\right|-R}
$$


Inequality (18) follows from the inequality

$$
n-k \geqslant(R+1) \sum_{j=1}^{p} \frac{k_{j}}{\left|z_{p}\right|-R}=\frac{k(R+1)}{\left|z_{p}\right|-R} .
$$

Let us check the validity of (19) for $R \in\left(1,\left(1-\frac{k}{n}\right)\left|z_{p}\right|-\frac{k}{n}\right)$. Since the function $\frac{R+1}{\left|z_{p}\right|-R}$ increases on $R$, it is sufficient to check (19) for $R=\left(1-\frac{k}{n}\right)\left|z_{p}\right|-\frac{k}{n}$. For such $R$, inequality (19) is

$$
(n-k)\left(\left|z_{p}\right|-\left(1-\frac{k}{n}\right)\left|z_{p}\right|+\frac{k}{n}\right) \geqslant k\left(\left(1-\frac{k}{n}\right)\left|z_{p}\right|-\frac{k}{n}+1\right) .
$$

Simple calculations show that (20) is an equality. Hence, $c_{p} \geqslant r_{p}$.

Remark. Note that Lemma 2 works only if $\left(1-\frac{k}{n}\right)\left|z_{p}\right|-\frac{k}{n}>1$, i. e.,

$$
\left|z_{p}\right|>\frac{n+k}{n-k}
$$

Now we have everything to obtain the following refinement of the Bernstein inequality (2):

Theorem 2. Let $0<r<1, f$ and $F$ be polynomials, such that

1) $\operatorname{deg} f \leqslant \operatorname{deg} F=n$;

2) all the zeros of $F$ belong to $\overline{\mathbb{D}}$; moreover, let $z_{1}, \ldots, z_{q}$ be all the zeros of $F$ of orders $k_{1}, \ldots, k_{q}, 1 \leqslant k_{1}+\cdots+k_{q} \leqslant n$, such that $r<\left|z_{q}\right|<\cdots<\left|z_{1}\right| \leqslant 1$

3) $|f(z)| \leqslant|F(z)|$ for $|z| \geqslant r$.

Then

$$
\left|f^{\prime}(z)\right| \leqslant\left|F^{\prime}(z)\right|
$$

for all $z,|z|=R$, where

$$
R \in\left[\left|z_{1}\right|, \infty\right) \cup\left(\bigcup_{m=1}^{q-1}\left(\left|z_{m+1}\right|,\left|z_{m}\right|\right) \backslash I_{m}\right) \cup\left(\left(r,\left|z_{q}\right|\right) \backslash I_{q}\right) .
$$

Here

$$
I_{1}=\left(\left(1-\frac{k_{1}}{n}\right)\left|z_{1}\right|-\frac{k_{1}}{n}\left|z_{2}\right|,\left(1-\frac{k_{1}}{n}\right)\left|z_{1}\right|+\frac{k_{1}}{n}\left|z_{2}\right|\right)
$$




$$
\begin{gathered}
I_{m}=\left(\left(1-\frac{k_{1}+\cdots+k_{m}}{n}\right)\left|z_{m}\right|-\frac{k_{1}+\cdots+k_{m}}{n}\left|z_{m+1}\right|,\left|z_{m}\right|\right), \\
m=2, \ldots, q-1, \\
I_{q}=\left(\left(1-\frac{k_{1}+\cdots+k_{q}}{n}\right)\left|z_{q}\right|-\frac{k_{1}+\cdots+k_{q}}{n} r,\left|z_{q}\right|\right) .
\end{gathered}
$$

Proof. 1) Firstly, consider the case $R \in\left[\left|z_{1}\right|,+\infty\right)$. Put $f_{1}(w)=f\left(\left|z_{1}\right| w\right)$, $F_{1}(w)=F\left(\left|z_{1}\right| w\right)$. For these polynomials, all assumptions of Theorem E are satisfied. Therefore, by Theorem E,

$$
\left|S_{\alpha}\left[f_{1}\right](w)\right| \leqslant\left|S_{\alpha}\left[F_{1}\right](w)\right|, \quad|w|=R_{1} \geqslant 1,
$$

where $\alpha \in \Omega_{R_{1}}$. This is equivalent to

$$
|w| z_{1}\left|f^{\prime}\left(\left|z_{1}\right| w\right)-\alpha n f\left(\left|z_{1}\right| w\right)\right| \leqslant|w| z_{1}\left|F^{\prime}\left(\left|z_{1}\right| w\right)-\alpha n F\left(\left|z_{1}\right| w\right)\right|,
$$

$|w|=R_{1} \geqslant 1, \alpha \in \Omega_{R_{1}}$. Denoting $z=w\left|z_{1}\right|, R=R_{1}\left|z_{1}\right|$, we have

$$
\left|S_{\alpha}[f](z)\right| \leqslant\left|S_{\alpha}[F](z)\right|, \quad|z|=R \geqslant\left|z_{1}\right|,
$$

for $\alpha \in \Omega_{\frac{R}{\left|z_{1}\right|}}$. Since $0 \in \Omega_{\frac{R}{\left|z_{1}\right|}}$, we obtain

$$
\left|f^{\prime}(z)\right| \leqslant\left|F^{\prime}(z)\right|, \quad|z|=R \geqslant\left|z_{1}\right| .
$$

2) Now let $R \in\left(\left|z_{2}\right|,\left|z_{1}\right|\right)$. The polynomials $f_{2}(w)=f\left(\left|z_{2}\right| w\right), F_{2}(w)=$ $=F\left(\left|z_{2}\right| w\right)$ satisfy the conditions of Lemma 1 , the polynomial $F_{2}$ has the unique zero $w=\frac{z_{1}}{\left|z_{2}\right|}$ outside $\overline{\mathbb{D}}$. The order of this zero equals $k_{1}$. By Lemma 1, $D\left(R_{2}, 1, n\right)$ contains 0 if

$$
R_{2} \in\left(1,\left|\frac{z_{1}}{z_{2}}\right|\right) \backslash\left(\left(1-\frac{k_{1}}{n}\right)\left|\frac{z_{1}}{z_{2}}\right|-\frac{k_{1}}{n},\left(1-\frac{k_{1}}{n}\right)\left|\frac{z_{1}}{z_{2}}\right|+\frac{k_{1}}{n}\right) .
$$

Therefore,

$$
\left|f_{2}^{\prime}(w)\right| \leqslant\left|F_{2}^{\prime}(w)\right|, \quad|w|=R_{2}
$$

for these $R_{2}$. Denoting $z=w\left|z_{2}\right|, R=R_{2}\left|z_{2}\right|$, rewrite (22) in the form $\left|f^{\prime}(z)\right| \leqslant\left|F^{\prime}(z)\right|$. Then the last inequality takes place for $|z|=R \in$ $\in\left(\left|z_{2}\right|,\left|z_{1}\right|\right) \backslash I_{1}$.

3) If $R \in\left(\left|z_{m+1}\right|,\left|z_{m}\right|\right), m=2, \ldots, q-1$, then we consider the following polynomials: $f_{m+1}(w)=f\left(\left|z_{m+1}\right| w\right)$ and $F_{m+1}(w)=F\left(\left|z_{m+1}\right| w\right)$. They satisfy the conditions of Lemma 2 with $p=m$. The polynomial $F_{m}$ 
has $m$ zeros $\frac{z_{1}}{\left|z_{m+1}\right|}, \ldots, \frac{z_{m}}{\left|z_{m+1}\right|}$ of orders $k_{1}, \ldots, k_{m}$ outside $\overline{\mathbb{D}}$. Applying Lemma 2, we obtain

$$
\left|f_{m+1}^{\prime}(w)\right| \leqslant\left|F_{m+1}^{\prime}(w)\right|, \quad|w|=R_{m+1}
$$

for

$$
R_{m} \in\left(1,\left|\frac{z_{m}}{z_{m+1}}\right|\right) \backslash\left(\left(1-\frac{k_{1}+\cdots+k_{m}}{n}\right)\left|\frac{z_{m}}{z_{m+1}}\right|-\frac{k_{1}+\cdots+k_{m}}{n} ;+\infty\right) .
$$

Denote $z=w\left|z_{m+1}\right|, R=R_{m+1}\left|z_{m+1}\right|$; from (23) we obtain $\left|f^{\prime}(z)\right| \leqslant$ $\leqslant\left|F^{\prime}(z)\right|$ for $|z|=R$, where $R \in\left(\left|z_{m+1}\right|,\left|z_{m}\right|\right) \backslash I_{m}$.

4) For $R \in\left(r,\left|z_{q}\right|\right)$ we repeat the reasonings from the previous step for the polynomials $f_{r}(w)=f(r w)$ and $F_{r}(w)=F(r w)$, and obtain the Bernstein inequality for $R \in\left(r ;\left|z_{q}\right|\right) \backslash I_{q}$.

Remark. If polynomials $f$ and $F$ satisfy the conditions of Theorem 2 and the inequality

$$
\min \left\{\left|\frac{z_{1}}{z_{2}}\right|,\left|\frac{z_{2}}{z_{3}}\right|, \ldots,\left|\frac{z_{q-1}}{z_{q}}\right|,\left|\frac{z_{q}}{r}\right|\right\}>\frac{n+k_{1}+\cdots+k_{q}}{n-k_{1}-\cdots-k_{q}}
$$

takes place, then (21) implies that inequality (2) is true not only in the complement to $\mathbb{D}$, but also in the following rings that are in $\mathbb{D}$ :

$$
\begin{gathered}
\Gamma_{1}=\left\{z \in \mathbb{C}:\left(1-\frac{k_{1}}{n}\right)\left|z_{1}\right|+\frac{k_{1}}{n}\left|z_{2}\right| \leqslant|z|<1\right\}, \\
\Gamma_{2}=\left\{z \in \mathbb{C}:\left|z_{2}\right|<|z| \leqslant\left(1-\frac{k_{1}}{n}\right)\left|z_{1}\right|-\frac{k_{1}}{n}\left|z_{2}\right|\right\}, \\
\Gamma_{q}=\left\{z \in \mathbb{C}:\left|z_{q}\right|<|z| \leqslant\left(1-\frac{k_{1}+\ldots+k_{q-1}}{n}\right)\left|z_{q-1}\right|-\frac{k_{1}+\cdots+k_{q-1}}{n}\left|z_{q}\right|\right\}, \\
\Gamma_{q+1}=\left\{z \in \mathbb{C}: r<|z| \leqslant\left(1-\frac{k_{1}+\cdots+k_{q}}{n}\right)\left|z_{q}\right|-\frac{k_{1}+\cdots+k_{q}}{n} r\right\} .
\end{gathered}
$$

Acknowledgments. The authors wish to express gratitude to the referee for careful reading of the manuscript and useful remarks. 


\section{References}

[1] Bernstein S. On the best approximation of continuous functions by polynomials of given degree. Typography and lithography of Zilberberg and sons, Kharkov, 1912 (in Russian).

Sur l'ordre de la meilleure approximation des functions continues par des polynomes de degré donné. Mémoires de la Classe des sciences. Académie royale de Belgique. Deuxiéme série. Tome IV, 1912 (in French).

[2] Bernstein S. Sur la limitation des dérivées des polynomes. C. R. Math. Acad. Sci. Paris, 1930, vol. 190, pp. 338-341. (in French)

[3] Bernstein S. N. Collected works. Vol. 1. Constructive functions theory (1905-1930). Izd. AN SSSR, Moscow, 1952 (in Russian).

[4] Ganenkova E. G., Starkov V. V. The Möbius transformation and Smirnov's Inequality for Polynomials. Mat. Zametki, 2019, vol. 105, no. 2, pp. $228-$ 239 (in Russian). DOI: https://doi.org/10.4213/mzm11858

Math. Notes, 2019, vol. 105, no. 2, pp. 216-226.

DOI: https://doi.org/10.1134/S0001434619010243

[5] Ganenkova E. G., Starkov V. V. Variations on a theme of the Marden and Smirnov operators, differential inequalities for polynomials. J. Math. Anal. Appl., 2019, vol. 476, is. 2, pp. 696-714.

DOI: https://doi.org/10.1016/j.jmaa.2019.04.006

[6] Goncharov V. L. Theory of the best approximation of functions. Scientific heritage of P. L. Chebyshev. Is.1. Mathematics Izd. AN SSSR, Moscow, Leningrad, 1945 (in Russian).

[7] Kompaneets E., Starkov V. Generalization of the Smirnov Operator and Differential Inequalities for Polynomials. Lobachevskii J. Math., 2019, vol. 40, no. 12 , pp. $2043-2051$.

DOI: https://doi.org/10.1134/S1995080219120047

[8] Kompaneets E. G., Starkov V. V. On the Smirnov type inequality for polynomials. Math. Notes, 2022 (to appear).

[9] Markov A. A. On a problem posed by D. I. Mendeleev. Izv. Akad. Nauk. St. Petersburg, 1889, vol. 62, pp. 1-24. (in Russian)

[10] Markov A. A. Selected works on theory of continued fractions and theory of functions deviating least from zero. OGIZ, Moscow, Leningrad, 1948 (in Russian).

[11] Markov V. A. On functions that deviates least from zero at the given interval. Tip. Imperatorskoi AN, St. Petersburg, 1892 (in Russian).

Markoff W. A. Über Polynome die in einen gegebenen Intervalle möglichst wenig von Null adweichen. Math. Ann., 1916, vol. 77, pp. 213-258 (in German). 
[12] Mendeleev D. I. Investigation of aqueous solutions by specific gravity. Tip. V. Demakova, St. Petersburg, 1887 (in Russian).

[13] Pommerenke Ch. On the derivative of a polynomial. Michigan Math. J., 1959, vol. 6 , no. 4 , pp. $373-375$.

DOI: https://doi.org/10.1307/mmj/1028998284

[14] Rahman Q. I., Schmeisser G. Analytic theory of polynomials. Oxford University Press, New York, 2002.

[15] Smirnoff V. I. Sur quelques polynomes aux propriétés extrémales. Transactions of the Kharkov mathematical society, 1928, vol. 4, no. 2, pp. 67-72 (in French).

[16] Smirnov V. I., Lebedev N. A. Constructive theory of functions of a complex variable. Nauka, Moscow, Leningrad, 1964 (in Russian). M.I.T. Press, Massachusetts Institute of Technology, Cambridge, MA, 1968.

[17] Steffens K.-G. The history of Approximation theory. From Euler to Bernstein. Birkenhaüser, Boston, Basel, Berlin, 2006.

[18] Szegö G. Über einen Satz von A. Markoff. Math. Z., 1925, vol. 23, pp. $45-$ 61 (in German).

[19] Wali S. L., Shah W. M., Liman A. Inequalities concerning B-operators. Probl. Anal. Issues Anal., 2016, vol. 5 (23), no. 1, pp. 55-72.

DOI: https://doi.org/10.15393/j3.art.2016.3250

Received September 11, 2021.

In revised form, September 28, 2021.

Accepted October 23, 2021.

Published online November 2, 2021.

Petrozavodsk State University

33 Lenina pr., Petrozavodsk 185910, Russia

E-mail: g_ek@inbox.ru, VstarV@list.ru 